\title{
Route Discovery and Capacity of Ad hoc Networks
}

\author{
Eugene Perevalov, Rick S. Blum, Xun Chen Anthony Nigara \\ Lehigh University, Bethlehem, PA 18015
}

\begin{abstract}
Throughput capacity of large ad hoc networks has been shown to scale adversely with the size of network $n$. However the need for the nodes to find or repair routes has not been analyzed in this context. In this paper, we explicitly take route discovery into account and obtain the scaling law for the throughput capacity under general assumptions on the network environment, node behavior, and the quality of route discovery algorithms. We also discuss a number of possible scenarios and show that the need for route discovery may change the scaling for the throughput capacity dramatically.
\end{abstract}

Keywords: Wireless networks, ad hoc networks, route discovery, throughput.

\section{INTRODUCTION}

The subject of this paper is the effect of the route discovery process $(R D P)$ on the throughput capacity of ad hoc networks. Previous results for the network capacity and throughput, like those found in [1]- [4] (see also [5][9] for an analysis of the effect of mobility on throughput), ignore the route discovery process and focus solely on the data traffic that ad hoc networks can support. On the other hand, under certain conditions (nodes leaving and joining, for example) the route discovery process can consume a significant portion of network resources and become detrimental to overall network performance and stability. For example, if more route discovery processes are initiated than can be sustained, then they will likely fail resulting in more retransmissions. In this scenario, the network can become inundated with route request $(R R E Q)$ packets and the overall network throughput can significantly decrease.

In the following, we determine the impact of the route discovery process on network throughput by determining the asymptotic behavior and scalability with the number of nodes for a network that has both data and RDP transmissions. Let $W$ be the number of bits that a node can successfully transmit per unit time. We characterize the throughput in terms of two additional basic $R D P$ related quantities:

- The average time that a route stays intact once established: $\tau(n)$.

- The function $G(\cdot)$ defined by $Q=G(f)$, where $Q$ is the probability that an $R D P$ is successful, and $f$ is the fraction of all nodes reached by that $R D P$.

We show that two qualitatively different situations can be distinguished.

1) $\tau(n) G\left(\frac{1}{n}\right)=o\left(\frac{1}{\sqrt{n \log n}}\right)$. In this case, the $R D P$ resource usage is severe enough to become the throughput bottleneck and change its scaling compared to the case when all routes are known. The throughput scales as

$$
\mathcal{T}(n)=\Theta\left(W \tau(n) G\left(\frac{1}{n}\right)\right) .
$$

2) $\tau(n) G\left(\frac{1}{n}\right)=\Omega\left(\frac{1}{\sqrt{n \log n}}\right)$. In this case the $R D P$ does not affect the throughput significantly (in the order of magnitude sense) and the main limiting factor for the throughput is still the interference between data transmissions:

$$
\mathcal{T}(n)=\Theta\left(\frac{W}{\sqrt{n \log n}}\right) .
$$

We apply these general results to some canonical examples, with some specific but reasonable assumed models for $\tau(n)$ and $G\left(\frac{1}{n}\right)$, to first show that the actual scaling of the throughput can be changed from the case where routing is ignored. In fact, for two of these cases we show

$$
\mathcal{T}(n)=\Theta\left(\frac{W}{n}\right)
$$


which implies routing can cause even more severe throughput scaling problems in ad hoc networks. This occurs, for example, when new nodes join a network for which $\tau(n)$ is assumed constant with $n$. On the other hand, later examples indicate that extremely efficient route repair can lessen, and maybe even eliminate, the just mentioned additional scaling problems.

\section{System Model}

We consider a wireless ad hoc network with $n$ nodes distributed uniformly over a unit square area. A node $i$ can be in two states: state $D$ and state $N$. In the state $D$, it can transmit data to its current destination $d(i)$, and in state $N$ it can't transmit (for example, due to lack of route knowledge). Let us assume that

- Nodes constantly change their states from $D$ to $N$ and back.

- The length of a period during which a node stays in the state $D$ has an expected value of $\tau(n)$ which is assumed to be determined exogenously.

- The length of a period during which a node stays in the state $N$ has an expected value of $\xi(n)$ which is to be determined in the course of analysis.

The identity of the destination $d(i)$ for the node $i$ may change from one active period (when the node is in state $D$ ) to another. In the case that the identity of $d(i)$ changes, we assume that the new destination is selected randomly. We also assume that one node can't play the role of a destination for two different source nodes simultaneously.

When a node is in $N$ state, it tries to discover the route to its new destination. For that purpose it broadcasts $R R E Q$ packets. Let $S_{R R E Q}$ be the size (in bits) of a $R R E Q$ packet. Recall $W$ is the number of bits that a node can successfully transmit per unit time. This implies that the transmission of a $R R E Q$ packet can be effected in a time of

$$
\delta t=\frac{S_{R R E Q}}{W} .
$$

In the following we assume that all time is slotted with the slot size equal to $\delta t$. In any time slot a node can either (re)transmit a data packet of size equal to $S_{R R E Q}$ or (re)broadcast a $R R E Q$ packet.

If in a given time slot all nodes are transmitting data packets then transmission success is governed by the Protocol Model in which the transmission from node $i$ to node $j$ within distance of $r$ from $i$ is successful if and only if there is no other transmitting node $k$ within the distance of $(1+\Delta) r$ from $j$. Here $r$ is the common transmission range which cannot be less than $\sqrt{\frac{\log n}{\pi n}}$ to ensure that the network is connected with high probability [11].

If all nodes are (re)transmitting $R R E Q$ packets in a given time slot, we use the following model for capture success.

A node can be in either listening mode or transmitting mode in any time slot. A transmitting node may either be initiating its own $R D P$ or forwarding a $R R E Q$ packet for another node's $R D P$. A node will be in the listening mode only when it has no $R R E Q$ packets to broadcast. If a node has received a $R R E Q$ in the previous time slot devoted to $R D P$, it will broadcast it in the next such time slot. A node will not forward the same RREQ packet more than once. It is assumed that all nodes use the same transmission power for broadcasting and a transmission by a node can be received in a region of area $a$ around the node. Here $a \leq 1$, and we assume that $a(n)=\Theta\left(\frac{1}{n}\right)$ to model the situation in which the physical transmission range of a node does not depend on the number of nodes in the network 1 .

A node will successfully receive a $R R E Q$ packet if the SINR for that packet is larger than all others it receives during the same time slot. Suppose there are $n_{t}$ nodes in transmitting mode in the current time slot, denoted by $t_{i}, i=1,2, \cdots, n_{t}$. Then there are $n-n_{t}=n_{r}$ nodes in listening mode in the network, which we denote by $s_{j}$, $j=1,2, \cdots, n_{r}$. For node $s_{j}$, there are only 3 possible cases: i) if it is out of the transmission range of any $t_{i}$, it will not receive anything, and it will stay in listening mode until the next time slot; ii) if it is only within the transmission range of one $t_{i}$, it will receive the $R R E Q$ packet from $t_{i}$; iii) if it is within the transmission range of multiple $t_{i}$ 's, node $s_{j}$ will successfully receive the $R R E Q$ packet that has the highest SINR as described in the capture model.

For the goal of this paper, we do not need to consider the situation when both data and $R R E Q$ packet transmissions take place in the same time slot. A time multiplexing approach to be described later is employed.

\footnotetext{
${ }^{1}$ Indeed, if $a_{p h}$ is a physical transmission range, then, since we keep the system area equal to $1, a(n)=\frac{a_{p h}}{A(n)}$, where $A(n)$ is the system area in physical units. The statement now follows when we realize that $A(n)=\Theta(n)$.
} 
We assume that a node in state $N$ attempts to find a route. For this purpose, it initiates an $R D P$ at a rate $\nu$. In the following we will measure both time periods and rates in time slots of physical length $\delta t$ with $\delta t$ given by (1). This implies that the rate of $R D P$ initiation by any node does not exceed 1 :

$$
\nu \leq 1
$$

\section{RDP SUCCESS PROBABILITY}

The key measure of the effectiveness of a route discovery process is the probability that it succeeds in finding the route. So we have to be able to characterize the probability of success of an $R D P$ in the given environment. We will do it using the following definition.

Definition 1. Let $G(\cdot)$ be a monotonically increasing function on the interval $[0,1]$ such that $G(0)=0$ and $G(1)=1$. Then, if $f_{k}$ is the fraction of nodes that the RDP process $k$ has reached, the probability of a successful route discovery by the process $k$ is $Q_{k}=G\left(f_{k}\right)$.

When thinking of possible shapes of the function $G(\cdot)$, it is reasonable to assume that the $R D P$ processes are "totally random" in the worst case. In other words, it is reasonable to exclude cases in which the probability of a node finding its destination is lower than the fraction of all nodes reached by the corresponding $R D P$ process. The latter situation is in principle possible. For example consider the situation in which the new nodes join the network in locations that are correlated with the locations of the corresponding destinations. If the correlation is such that the average distance between the source and destination exceeds the average distance in the network, it is possible to have $G(f)<f$ for $0<f<1$. However it is fairly clear that such a situation is "unnatural" and we assume that nothing like this actually happens. With this assumption, we have the following.

Proposition 1. $G(f) \geq f$ for $0 \leq f \leq 1$.

Since, clearly, $G(0)=0$ and $G(1)=1$, it is also reasonable to assume that the function $G(f)$ is concave.

We also introduce an unconditional route discovery success probability $Q$ which we will treat as a function of the total $R D P$ arrival rate in the network $\lambda$. It turns out to be possible to put an upper bound on $Q$ in terms of $\lambda$ and the average number $\bar{n}_{r}$ of first-time $R R E Q$ packet receptions in a time slot. The proof of the next lemma is omitted due to lack of space.

Lemma 1. If the route discovery is described by the function $G(f)$, then the unconditional route discovery probability $Q$ is upper bounded as

$$
Q \leq G(\bar{f})=G\left(\frac{\bar{n}_{r}}{\lambda(n-1)}\right)
$$

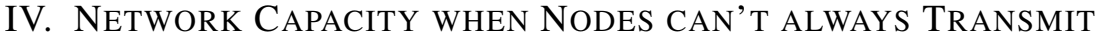

First, let us consider the case when, for large $n$, the average length of active periods (when nodes are in the $D$ state) is not much smaller than that of period of "dormancy" (when nodes are in the $N$ state). In the asymptotic notation, this means that

$$
\tau(n)=\Omega(\xi(n)) .
$$

In this case, it is easy to see that the results on capacity reported in [1] are valid.

Next, consider the case when the average length of active periods becomes negligible compared to the "dormant" ones as the network size $n$ increases, i.e.

$$
\tau(n)=o(\xi(n))
$$

in the asymptotic notation. For this case we have a different upper bound on the per node throughput of the network. To find it, we need an auxiliary result stated as a lemma whose proof is quite straightforward (see [16]) and is omitted here.

Lemma 2. The proportion of time spent by a node in the state $D$ is

$$
\frac{\tau(n)}{\tau(n)+\xi(n)} \text {. }
$$


With the above lemma, we can obtain a "dormancy induced" upper bound on the throughput.

Theorem 1. If every node alternates between states $D$ and $N$ spending an average of $\tau(n)$ time slots in state $D$ and an average of $\xi(n)$ time slots in state $N$ then the per node throughput is

$$
\mathcal{T}(n)=O\left(\frac{W \tau(n)}{\xi(n)}\right)
$$

Proof. Consider long time $T$ (measured in RDP time slots). According to Lemma 2, only $T \frac{\tau(n)}{\tau(n)+\xi(n)}$ of these time slots can be used by any node for data transmission. During these time slots a node can send no at most $T \frac{\tau(n)}{\tau(n)+\xi(n)} S_{R R E Q}$ bits to its destinations. So the inequality

$$
\mathcal{T}(n) T \delta t \leq T \frac{\tau(n)}{\tau(n)+\xi(n)} S_{R R E Q}
$$

has to hold. Since $\delta t=\frac{S_{R R E Q}}{W}$, we obtain from (2) that

$$
\mathcal{T}(n) \leq \frac{W \tau(n)}{\tau(n)+\xi(n)} \leq \frac{W \tau(n)}{\xi(n)},
$$

which proves the theorem.

On the other hand, regardless of states of nodes, we have the following upper bound on the throughput induced by interference between simultaneous data transmissions.

Theorem 2. The per node throughput $\mathcal{T}(n)$ is upper bounded as

$$
\mathcal{T}(n)=O\left(\frac{W}{\sqrt{n \log n}}\right) .
$$

The proof is standard in the literature on capacity of ad hoc networks and is omitted.

Combining Theorems 1 and 2, and choosing the tighter bound depending on the behavior of the ratio $\frac{\tau(n)}{\xi(n)}$ we obtain the following corollary.

Corollary 1. The per node throughut $\mathcal{T}(n)$ is upper bounded as

$$
\mathcal{T}(n)=O\left(\frac{W \tau(n)}{\xi(n)}\right)
$$

if $\frac{\tau(n)}{\xi(n)}=o(1 / \sqrt{n \log n})$ and it is upper bounded as

$$
\mathcal{T}(n)=O\left(\frac{W}{\sqrt{n}}\right)
$$

if $\left.\frac{\tau(n)}{\xi(n)}=\Omega(1 / \sqrt{n \log n})\right)$.

In order to show that the bounds of Corollary 1 are achievable up to constant we will demonstrate that there exists a feasible transmission schedule that allows us to obtain the required per node throughput. To achieve that goal, we tesselate the square region into identical square cells with side of size

$$
g(n)=\sqrt{\frac{2 \log n}{n}}
$$

Two cells are called interfering neighbors if there is a point in one cell within a distance of $(2+\Delta) r(n)$ from a point in the other cell. It is easy to see that only transmissions from the cells that are interfering neighbors can interfere with each other. The following two lemmas are standard in the literature on ad hoc network capacity (see for example [1]). 
Lemma 3. There exists a transmission schedule in which each cell can transmit in one of every $\tilde{c}+1$ time slots, where $\tilde{c}$ depends only on the parameter $\Delta$.

Lemma 4. The probability that there is a cell that does not contain a single node is upper bounded by

$$
\frac{1}{2 n \log n},
$$

In other words, all cells contain at least one node with high probability.

We organize transmission in the following way. The entire system is tesselated into square cells of area $g\left(\rho_{s}\right)^{2}$.

The routing of packet between nodes proceeds as follows. To route a packet between two nodes, we employ at most two straight lines: one vertical and one horizonta [2. Each time a packet is transmitted from a node in a cell to some node in an adjacent cell. In the final hop, the packet is transmitted to the destination from a node in a cell adjacent to the cell containing the destination.

Now, let us consider a given cell $C_{i}$ and count the number of routes passing through it. Let us denote this number by $N_{i}$. For $N_{i}$, we have the following two results the proof of which makes use of Chernoff bound and is fairly straightforward. It can be found in [16] and is omitted here due to size limitations.

\section{Lemma 5.}

$$
\max _{i} N_{i}=O(\sqrt{n \log n})
$$

with high probability.

\section{Lemma 6.}

$$
\min _{i} N_{i}=\Omega(\sqrt{n \log n})
$$

with high probability.

We can now find the achievable per node throughput. This is the subject of the next two theorems.

Theorem 3. If

$$
\frac{\tau(n)}{\xi(n)}=o\left(\frac{1}{\sqrt{n \log n}}\right)
$$

then the per node throughput

$$
\mathcal{T}(n)=\Omega\left(\frac{W \tau(n)}{\xi(n)}\right)
$$

is achievable with high probability.

Proof. Consider a long time $T$. Since each source can generate data only in the $D$ state, using Lemmas 2 and 5 , we see that the number of packets $N_{T, i}$ that has to be served by the cell $C_{i}$ can be upper bounded as

$$
N_{T, i} \leq \max _{i} N_{i} \frac{\tau(n)}{\xi(n)} T=c \sqrt{n \log n} \frac{\tau(n)}{\xi(n)} T
$$

with high probability. Since $\frac{\tau(n)}{\xi(n)}=o\left(\frac{1}{\sqrt{n \log n}}\right)$, we have that, with high probability,

$$
N_{T, i}=o(T),
$$

which is less than the number of time slots $\frac{T}{\tilde{c}}$ that, as shown in lemma 3 each cell can be active in. This implies that the per node throughput of

$$
\frac{\frac{\tau(n)}{\xi(n)} S_{R R E Q} T}{T \delta t}=\frac{\tau(n)}{\xi(n)} W
$$

is achievable with high probability, which proves the theorem.

The meaning of the next theorem is that, if the ratio $\frac{\tau(n)}{\xi(n)}$ is large enough, the throughput limited by the interference between data transmissions can be achieved. Its proof is similar to that of the previous theorem and is omitted.

\footnotetext{
${ }^{2}$ It is possible that only one straight line is needed
} 


\section{Theorem 4. If}

$$
\frac{\tau(n)}{\xi(n)}=\Omega\left(\frac{1}{\sqrt{n \log n}}\right)
$$

then the per node throughput

$$
\mathcal{T}(n)=\Omega\left(\frac{W}{\sqrt{n \log n}}\right)
$$

is achievable with high probability.

\section{SCALING of $\xi(n)$}

A node that needs to find a route will initiate an $R D P$. Since it may not be successful, the node might have to initiate it several times. We assume that the node initiates RDP's with frequency of $\nu$ until the route is found. The next lemma computes the expected number of RDP's that a node will need to initiate in order to find the route. The proof of the following lemma is elementary.

Lemma 7. The average number of RREQ transmissions, $N_{\text {avg, }}$, which is required by a node for a successful route discovery to the same destination, is given by

$$
N_{\text {avg }}=\frac{1}{Q}
$$

Let us introduce $Q^{\prime}(\lambda)$ as the unconditional probability for a success of an RDP in the presence of data transmission. Obviously, $Q^{\prime}(\lambda)$ may depend on how the network resources (time and space) are divided between $R D P$ and data transmission. It's clear that for any such scheme

$$
Q^{\prime}(\lambda) \leq Q(\lambda)
$$

The next lemma relates the frequency of $R D P$ initiation $\nu$, the average time of route life $\tau(n)$ and the total $R D P$ arrival rate $\lambda$.

Lemma 8. The total RDP arrival rate can be computed as

$$
\lambda=\frac{n \nu}{\nu \tau(n) Q^{\prime}(\lambda)+1}
$$

Proof. As shown in Lemma 7 a node needs to initiate on average $\frac{1}{Q^{\prime}(\lambda)} R D P$ 's for a successful route discovery. The time between two consecutive initiations is given by $\frac{1}{\nu}$. The total time of transmitting $\frac{1}{Q^{\prime}(\lambda)} R R E Q$ packets is then $\frac{1}{Q^{\prime}(\lambda) \nu}$. After this total time, the valid route is set up, and lasts for time $\tau(n)$ during which the node can transmit data and no route discovery is required.

Thus, on average, each node initiates $\frac{1}{Q^{\prime}(\lambda)} R R E Q$ packets every time period of $\frac{1}{Q^{\prime}(\lambda) \nu}+\tau(n)$. Therefore the $R D P$ arrival rate due to one node can be found as

$$
\frac{\lambda}{n}=\frac{\frac{1}{Q^{\prime}(\lambda)}}{\frac{1}{Q \nu}+\tau(n)}=\frac{\nu}{1+Q^{\prime}(\lambda) \tau(n) \nu},
$$

yielding,

$$
\lambda=\frac{n \nu}{1+Q^{\prime}(\lambda) \tau(n) \nu}
$$

for the total $R D P$ arrival rate.

We would like to demonstrate that the average length of a node "inactivity" period (when it is in the state $N$ attempting to discover a route) is bounded from below and the bound depends on the shape of the route discovery success function $G(\cdot)$.

We can now state the lower bound result.

Theorem 5. The expected length of the time interval during which a node stays in the $N$ state is

$$
\xi(n)=\Omega\left(\frac{1}{G\left(\frac{1}{n}\right)}\right) .
$$


The proof makes use of Lemmas 1 and 8 and is omitted due to lack of space.

Next, we would like to find an upper bound on the average length of "data inactivity" period $\xi(n)$. Note that, in order to find a lower bound, it was sufficient to assume that all network resources were devoted to route discovery with no data transmission taking place. For an upper bound, we need to present a constructive network resource division scheme between $R D P$ and data transmission.

We make the following assumption about the probability distribution of the fraction of nodes reached by an $R D P$. Assumption 1: The probability distribution of the fraction of nodes reached by an $R D P$ is such that $m_{f} \geq \gamma \bar{f}$, where $m_{f}$ is the median of the distribution, and $\gamma>0$ is a constant independent on $n$.

Note that the goal of making this assumption is to rule out "pathological" distributions of the fraction of nodes reached by an $R D P$. Thus it is not restrictive in that any distribution that can be realized in practice should satisfy it for an appropriate value of $\gamma$.

Let us adopt a time division scheme in which in any given time slot either only data transmission or only route discovery takes place. In other words, all time slots are divided into two types: data slots and $R D P$ slots. During data slots, only the data transmission takes place whereas during $R D P$ slots only $R D P$ processes are allowed to run. Let $\theta$ be the fraction of $R D P$ time slots. We call this resource division scheme "Scheme A".

Let us denote by $Q^{\prime}$ the success probability in the presence of data transmission. In the time division scheme that we are using, it is straightforward to find $Q^{\prime}(\lambda)$.

Lemma 9. Under Scheme A, the unconditional probability of route discovery success $Q^{\prime}(\lambda)$ can be found as $Q^{\prime}(\lambda)=Q\left(\frac{\lambda}{\theta}\right)$.

With the new success probability $Q^{\prime}(\lambda)$ is is straightforward to find the relation between the rate of retrial for every node $\nu$ and the total arrival rate $\lambda$. For this we just need to repeat the steps that led to the statement of lemma 8 but with $Q^{\prime}$ instead of $Q$. We state the result as a lemma.

Lemma 10. Under the time division Scheme A, the following relation holds between $\nu$ and $\lambda$.

$$
\lambda=\frac{n \nu}{\nu \tau Q^{\prime}(\lambda)+1} .
$$

To derive an upper bound on the average length $\xi(n)$ of "data inactivity" period, we need the following auxiliary results whose proof is omitted due to lack of space.

\section{Lemma 11.}

$$
\frac{\bar{n}_{r}}{n} \geq \hat{c},
$$

where $\hat{c}$ is a constant independent on $n$.

The next lemma puts a lower bound on the unconditional probability of success of an RDP under Assumption 1.

Lemma 12. If Assumption 1 holds, then $Q(\lambda) \geq \frac{1}{2} G\left(\frac{\gamma \bar{n}_{r}}{\lambda n}\right)$.

Proof. Since $Q(\lambda)=\overline{G(f)}$, by definition of a median, we have

$$
Q(\lambda)=\overline{G(f)} \geq \frac{1}{2} G\left(m_{f}\right) \geq \frac{1}{2} G(\gamma \bar{f})=\frac{1}{2} G\left(\frac{\gamma \bar{n}_{r}}{\lambda n}\right),
$$

which proves the lemma.

We can now use the above lemmas to find an upper bound on $\xi(n)$.

Theorem 6. Under scheme A

$$
\xi(n)=O\left(\frac{1}{G\left(\frac{1}{n}\right)}\right) .
$$

The proof of this theorem follows in a straightforward fashion from Lemmas 11 and 12 and is omitted due to size limitations. 
Putting together the results of Theorems 5 and 6 , we obtain a tight asymptotic characterization of the quantity $\xi(n)$ which we state as a corollary.

\section{Corollary 2.}

$$
\xi(n)=\Theta\left(\frac{1}{G\left(\frac{1}{n}\right)}\right)
$$

\section{RDP LIMITED THROUGHPUT}

In this section, we collect the pieces to obtain the main result of this paper: the scaling of the $R D P$ limited throughput of a random ad hoc network. The next theorem covers the case where the RDP plays the role of the throughput bottleneck.

Theorem 7. If $\tau(n) G\left(\frac{1}{n}\right)=o\left(\frac{1}{\sqrt{n \log n}}\right)$ then

$$
\mathcal{T}(n)=\Theta\left(W \tau(n) G\left(\frac{1}{n}\right)\right) .
$$

Proof. If $\tau(n) G\left(\frac{1}{n}\right)=o\left(\frac{1}{\sqrt{n \log n}}\right)$ then, according to Corollary 2 ,

$$
\frac{\tau(n)}{\xi(n)}=o\left(\frac{1}{\sqrt{n \log n}}\right) \text {. }
$$

On the other hand, combining the results of Theorems 1 and 3 , we obtain that if $\frac{\tau(n)}{\xi(n)}=o\left(\frac{1}{\sqrt{n \log n}}\right)$, then

$$
\mathcal{T}(n)=\Theta\left(\frac{W \tau(n)}{\xi(n)}\right)
$$

and therefore (Corollary 2)

$$
\mathcal{T}(n)=\Theta\left(W \tau(n) G\left(\frac{1}{n}\right)\right)
$$

which proves the theorem.

The following theorem shows that when the product $\tau(n) G\left(\frac{1}{n}\right)$ is large enough, the main limiting factor for the throughput is the interference between simultaneous data transmissions and we are back to the case described in [1].

Theorem 8. If $\tau(n) G\left(\frac{1}{n}\right)=\Omega\left(\frac{1}{\sqrt{n \log n}}\right)$ then

$$
\mathcal{T}(n)=\Theta\left(\frac{W}{\sqrt{n \log n}}\right)
$$

Proof. If $\tau(n) G\left(\frac{1}{n}\right)=\Omega\left(\frac{1}{\sqrt{n \log n}}\right)$ then, according to Corollary 2 ,

$$
\frac{\tau(n)}{\xi(n)}=\Omega\left(\frac{1}{\sqrt{n \log n}}\right) \text {. }
$$

Now, combining the results of Theorems 2 and 4, we see that if $\frac{\tau(n)}{\xi(n)}=\Omega\left(\frac{1}{\sqrt{n \log n}}\right)$, then

$$
\mathcal{T}(n)=\Theta\left(\frac{W}{\sqrt{n \log n}}\right)
$$

which proves the theorem.

Now that the general asymptotic behavior of the $R D P$ limited throughput is given, let us consider several simple examples and obtain the throughput scaling. 
Examples:

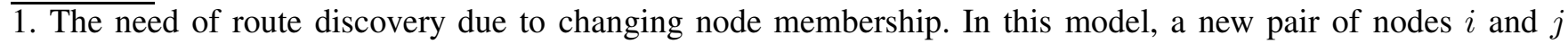
join the network and need to discover a route to each other. They stay connected during a time period of $\tau_{i j}$ after which the connection is terminated and the nodes leave the network (turn themselves off). This process of pairs joining the network and leaving continues is such a way that the network is in an equilibrium in the sense of the number of nodes participating in it at any given time. Let us assume that $E\left(\tau_{i j}\right)=\tau$ for any $i$ and $j$. In this case it is reasonable to assume that $\tau$ does not depend on $n$, as it depends only on the behavior pattern of individual nodes. Since both nodes that just have joined the network are "new" to it, the RDP success probability function is $G(f)=f$. This implies that

$$
G\left(\frac{1}{n}\right)=\Theta\left(\frac{1}{n}\right)
$$

and therefore,

$$
\tau(n) G\left(\frac{1}{n}\right)=\Theta\left(\frac{1}{n}\right)=o\left(\frac{1}{\sqrt{n \log n}}\right) .
$$

Then it follows form Theorem 7 that the RDP limited throughput scales as

$$
\mathcal{T}(n)=\Theta\left(\frac{W}{n}\right)
$$

2. The node membership in the network is constant but the loss of routes is due to severe fading or excessive node mobility. In this case, it would be reasonable to assume that a route from a node $i$ to $d(i)$ is lost and has to be rediscovered whenever one (or another constant number) link is broken. The number of links between $i$ and $d(i)$ is $\Theta\left(\frac{1}{\sqrt{n}}\right)$, and the rate at which a link brakes depends only on fading environment and the mobility characteristics and therefore is independent on $n$. Therefore, assuming that the links brake independently, we obtain that the rate at which the nodes $i$ and $d(i)$ lose their route will be $\Theta\left(\frac{1}{\sqrt{n}}\right)$. Hence, the average length of time during which the route stays intact is

$$
\tau(n)=\Theta\left(\frac{1}{\sqrt{n}}\right) .
$$

On the other hand, since both the source and the destination are still present in the network, they are "known" to $\Theta(\sqrt{n})$ other nodes (the number of routes passing through a typical node). This means that in order to discover a route to the destination, an $R D P$ initiated by the source needs to reach one of $\Theta(\sqrt{n})$ nodes in the network. Therefore,

$$
G\left(\frac{1}{n}\right)=\Theta\left(\frac{1}{\sqrt{n}}\right),
$$

and hence (Theorem 7),

$$
\mathcal{T}(n)=\Theta\left(\frac{W}{n}\right) .
$$

3. The situation is just as above with the exception of the route discovery probability. Assume that the route repair algorithm is so good that it is able to repair the broken link immediately so that the CL model is appropriate. Then

$$
\tau(n)=\Theta\left(\frac{1}{\sqrt{n}}\right),
$$

as above, but

$$
G\left(\frac{1}{n}\right)=\Theta(1)
$$

In this case, Theorem 8 yields

$$
\mathcal{T}(n)=\Theta\left(\frac{W}{\sqrt{n \log n}}\right) .
$$

In other words, the scaling is the same as in the case with no route discovery meaning that under such conditions the main limitation is still data transmission. 


\section{REFERENCES}

[1] P. Gupta And P.R. Kumar, The Capacity of Wireless Networks, IEEE Transactions on Information Theory, IT-46, pp. 388-404, March 2000.

[2] P. Gupta And P.R. Kumar, Towards an Information Theory of Large Networks: an Achievable Rate Region, In Proceedings of 2001 IEEE International Symposium on Information Theory, (2001) 159.

[3] L-L. XIE AND P. R. KUMAR, A network information theory for wireless communication: scaling laws and optimal operation, IEEE Transactions on Information Theory, vol 50, no. 5, pp. 748-767, May 2004.

[4] S. Toumpis And A. Goldsmith, Capacity regions for wireless ad hoc networks, IEEE Transactions on Wireless Communications, 2(4), (2003) p. 736.

[5] M. Grossglauser And D. Tse, Mobility Increases the Capacity of Ad Hoc Wireless Networks, In Proceedings of INFOCOM 2000, 3 (2000) 1360.

[6] S.N. Diggavi, M. Grossglauser And D. Tse, Even one-dimensional mobility increases ad hoc wireless capacity, in Proceedings of 2002 IEEE International Symposium on Information Theory, p. 352.

[7] N. BASAl AND Z. LiU, Capacity, delay and mobility in wireless ad hoc networks, in Proceedings of INFOCOM 2003, 2, pp. 1553 1563.

[8] E. Perevalov and R. Blum, Delay limited throughput of ad hoc networks, IEEE Transactions on Communications, 52, pp. 1957 1968.

[9] A. El Gamal, J. Mammen, B. Prabhakar and D. Shan, Throughput-delay trade-off in wireless networks, In Proceedings of INFOCOM 2004.

[10] X. Chen, C. C. ChAt, Y. H. Chew, Performance of adaptive MQAM in cellular system with Nakagami fading and log-normal shadowing, In Proceeding of IEEE 58th Vehicular Technology Conference. VTC 2003-Fall, pp. 1549-1553 Vol.3.

[11] P. GUPTA AND P.R. KUMAR, Critical power for asymptotic connectivity in wireless networks, in Stochastic Analysis, Control, Optimization nad Applications: A Volume in Honor of W.H. Fleming, W.M. McEneany, G. Yin and Q. Zhang, eds., Boston, MA, Birkhauser, 1998.

[12] X. Chen, C. C. Chat, Y. H. CHEw, Constrained power allocation algorithm for rate adaptive MIMO system, In Proceeding of Eighth IEEE International Symposium on Spread Spectrum Techniques and Applications, 2004, pp. 924-928.

[13] A. Agarwal AND P.R. KumaR, Improved capacity bounds for wireless networks, Wireless Communications and Mobile Computing, vol. 4, pp. 251-261, 2004.

[14] C. C. Chat, X. Chen, Y. H. Chew, Power control for minimum outage in interference-limited Nakagami fading wireless channels, In 14th IEEE Proceedings on Personal, Indoor and Mobile Radio Communications, 2003. PIMRC 2003., vol 2, pages $1274-1278$.

[15] E. Perevalov, R. Blum, X. Chen, And A. Nigara, An analysis of the route discovery dynamics in wireless ad hoc networks, submitted for journal publication.

[16] E. Perevalov, R. Blum, X. Chen, And A. Nigara, The Impact of Route Discovery on the Throughput of Wireless Ad Hoc Networks, submitted for journal publication. 